\title{
Ray-tracing-based reconstruction algorithms for digital breast tomosynthesis
}

\author{
Weihua Zhou, ${ }^{a}$ Jianping Lu, ${ }^{b, c}$ Otto Zhou, ${ }^{b, c}$ and Ying Chen ${ }^{a, *}$ \\ aSouthern Illinois University, Department of Electrical and Computer Engineering, 1230 Lincoln Drive, Mail Code 6603, Carbondale, \\ Illinois 62901, United States \\ bUniversity of North Carolina, Department of Physics and Astronomy, and Curriculum in Applied Sciences and Engineering, \\ 120 E. Cameron Avenue, Chapel Hill, North Carolina 27599-3255, United States \\ 'University of North Carolina, Lineberger Comprehensive Cancer Center, School of Medicine, Chapel Hill, North Carolina 27599-7295, \\ United States
}

\begin{abstract}
As a breast-imaging technique, digital breast tomosynthesis has great potential to improve the diagnosis of early breast cancer over mammography. Ray-tracing-based reconstruction algorithms, such as ray-tracing back projection, maximum-likelihood expectation maximization (MLEM), ordered-subset MLEM (OS-MLEM), and simultaneous algebraic reconstruction technique (SART), have been developed as reconstruction methods for different breast tomosynthesis systems. This paper provides a comparative study to investigate these algorithms by computer simulation and phantom study. Experimental results suggested that, among the four investigated reconstruction algorithms, OS-MLEM and SART performed better in interplane artifact removal with a fast speed convergence. (C) 2015 SPIE and IS\&T [DOI: 10.1117/1.JEI.24.2.023028]
\end{abstract}

Keywords: digital breast tomosynthesis; reconstruction algorithm; ray-tracing.

Paper 14401 received Jul. 24, 2014; accepted for publication Mar. 18, 2015; published online Apr. 15, 2015.

\section{Introduction}

Breast cancer is the second-leading cause of cancer-related deaths among women in the United States. Approximately one in eight women in USA will be diagnosed with breast cancer during their lifetime. ${ }^{1}$ Mammography is the most effective tool for the early detection of breast cancer among current breast-imaging techniques. ${ }^{1}$ However, the overlapping of breast tissues in two-dimensional (2-D) imaging makes it difficult to interpret the images. ${ }^{2} \mathrm{~A}$ breast cancer can be hidden in the overlapping tissues and not be detected. Breast tomosynthesis may allow us to detect smaller lesions that would be hidden with standard mammograms. ${ }^{2,3}$ Researchers believe that this new imaging technique is promising to replace mammography imaging. ${ }^{2}$

Tomosynthesis reconstruction algorithms transform 2-D projection images into a three-dimensional (3-D) volume. A commonly used reconstruction model is to divide the breast volume into a great number of small nonoverlapping voxels with constant attenuation coefficients which represent how easily the material can be penetrated by an x-ray beam. $^{4,5}$ When an x-ray projection line penetrates these voxels, the ray-tracing model is used to calculate the x-ray attenuation along the path of the projection line. With assumptions of the relationship between the incident and detected x-ray intensities, the ray-tracing model, in practice, has been used in several reconstruction algorithms, such as simultaneous algebraic reconstruction technique $\left(\right.$ SART) ${ }^{6}$ and maximum-likelihood expectation maximization (MLEM), ${ }^{7,8}$ to reconstruct 3-D images. These algorithms have been developed and implemented in the design of several breast tomosynthesis prototype systems. ${ }^{6-8}$

\footnotetext{
*Address all correspondence to: Ying Chen, E-mail: adachen@ @iu.edu
}

Consequently, it is important to investigate the ray-tracing-based reconstruction algorithms and to evaluate the image quality. In our previous work, a sparse matrix method was used to improve the ray-tracing model and speed up the MLEM reconstruction algorithm. ${ }^{9}$ This paper presents our investigation about the convergence and image quality of inplane sharpness and interplane blurring removal of the reconstruction algorithms using the ray-tracing model. Four representative ray-tracing-based reconstruction algorithms, including ray-tracing back projection (ray-tracing BP), ${ }^{6}$ MLEM, ${ }^{7,8}$ ordered-subset MLEM (OS-MLEM), ${ }^{10}$ and SART ${ }^{6}$ were evaluated by computer simulation and phantom study.

\section{Methods}

\subsection{Ray-Tracing Model}

The ray-tracing model divides the object into small voxels with a constant attenuation coefficient $\mu$. The intensity attenuation can be written as ${ }^{5}$

$O=I \cdot e^{-\sum_{i}(l \cdot \mu)}$

where $O$ is the detected x-ray intensity, $I$ is the incident x-ray intensity, $i$ is the voxel which is penetrated by the projection line, $\mu$ is the attenuation coefficient, and $l$ is the path length where the x-ray projection line passes through the voxel.

Equation (1) can be rewritten as a linear form

$\sum_{i}(l \cdot \mu)=A_{i}$

where $A_{i}=\log (I / O)$.

1017-9909/2015/\$25.00 @ 2015 SPIE and IS\&T 
Only a few voxels are penetrated by each projection line, and thus a data structure based on the sparse matrix was used to accelerate the MLEM algorithm in our previous work. ${ }^{9}$ This technique was used for the investigation of the following four reconstruction algorithms.

\subsection{Ray-Tracing Back Projection}

In the linear attenuation Eq. (2), the total intensity attenuation depends on both attenuation coefficients as well as the path lengths. The ray-tracing BP was proposed based on this fact. ${ }^{6}$ It can be written as

$\mu_{j}=\frac{\sum_{i=1}^{M} l_{i j}\left(\frac{A_{i}}{L_{i}}\right)}{\sum_{i=1}^{M} l_{i j}}$.

This equation takes into account the proportion of individual path lengths. $i$ is the $i$ 'th projection line, $j$ is the $j$ 'th voxel and $L_{i}$ is the total path of the $i$ 'th projection line.

\subsection{Maximum-Likelihood Expectation Maximization}

MLEM attempts to maximize the likelihood of getting the detected x-ray intensity from the incident intensity and $\mathrm{x}$-ray attenuation model. It assumes that the statistical relationship between the detected and incident $\mathrm{x}$-ray follows the Poisson distribution. ${ }^{11,12}$

The attenuation coefficient $\mu$ can be acquired by an iterative equation ${ }^{6-8}$

$\mu_{j}^{(t+1)}=\mu_{j}^{(t)}+\Delta \mu_{j}^{(t)}=\mu_{j}^{(t)}+\frac{\mu_{j}^{(t)} \sum_{i} l_{i j}\left(I_{i} e^{-\sum_{i} l_{i j} \mu_{j}^{(t)}}-O_{i}\right)}{\sum_{i}\left(l_{i j}\left\langle l, \mu^{(t)}\right\rangle_{i} I_{i} e^{-\sum_{i} l_{i j} \mu_{j}^{(t)}}\right)}$,

where $O_{i}$ is the detected x-ray intensity pixel value for the $\mathrm{x}$-ray projection line $i . i$ is the individual voxel in the $3-\mathrm{D}$ attenuation model. $I_{i}$ is the incident x-ray intensity to the pixel $i . l_{i j}$ is the path length of the intersection between the voxel $j$ and the $x$-ray projection line from the $x$-ray source to the pixel $i$ on the detector.

\subsection{Ordered-Subset Maximum-Likelihood Expectation Maximization}

The MLEM converges very slowly due to its absorption of all the projection information in each iteration. OS-MLEM provides the order-of-magnitude acceleration over MLEM. ${ }^{10}$ In OS-MLEM, only a subset of the projection image dataset is used for each iteration.

In our implementation, we used a direct transform of MLEM and changed the original update into a projectionby-projection view update. To improve the convergence, we designed a special update order to maximize the angle separation ${ }^{13}$ between the successive absorbed projection views.

\subsection{Simultaneous Algebraic Reconstruction Algorithm}

In algebraic iterative reconstruction algorithms, ${ }^{14}$ the procedure of estimating the attenuation coefficients, generating a new set of projection images from the estimate, comparing the simulated images to real projection data, and then smearing the difference back to generate a new estimate, is iteratively called.

In our SART implementation, the solution for the attenuation coefficients is expressed as ${ }^{6}$

$\mu_{j}^{(t+1)}=\mu_{j}^{(t)}+\Delta \mu_{j}^{(t)}=\mu_{j}^{(t)}+\frac{\left.\sum_{i} l_{i j} \frac{A_{i}-\sum_{j} l_{i j} \mu_{j}^{t}}{\sum_{j} l_{i j}}\right)}{\sum_{i} l_{i, j}}$.

\section{Experiments}

A multibeam Deutsche Bundespost Telekom prototype system built up by our collaborators ${ }^{15-18}$ was used to generate the tomosynthesis projection images. Figure 1 illustrates parallel-imaging geometry in the imaging system. Multiple $\mathrm{x}$-ray sources are aligned along the $x$ direction that is parallel to the detector plane. This system has 31 evenly distributed $\mathrm{x}$-ray beam sources. The vertical distance from the x-ray tubes to the detector (source-to-image distance) is $692.8 \mathrm{~mm}$ in our experiments. A digital flat-panel detector with a pixel size of $140 \mu \mathrm{m}$ is integrated into the system.

\subsection{Sphere Simulation}

A solid sphere was simulated with a different attenuation coefficient of $0.038 \mathrm{~mm}^{-1}$ to imitate the mass. ${ }^{19}$ An finite element modeling (FEM) method was used to create the spherical object and then to generate the projection images. The reconstruction algorithms of the ray-tracing BP, MLEM, OS-MLEM, and SART were used to reconstruct the spherical object. Then, both the attenuation error and mean square error (MSE) were calculated.

The attenuation error was calculated based on Eq. (1), i.e.,

Attenuation error $=\sum_{i}\left|O_{i}-I_{i} \cdot e^{-\sum_{i}(l \cdot u)}\right|$,

for all the X-ray projection lines.

The MSE between the real finite elements and the reconstructed volume was defined as

$\operatorname{MSE}=\frac{\sum_{j=1}^{J}\left|\mu_{j}-\hat{\mu}_{j}\right|}{J}$,

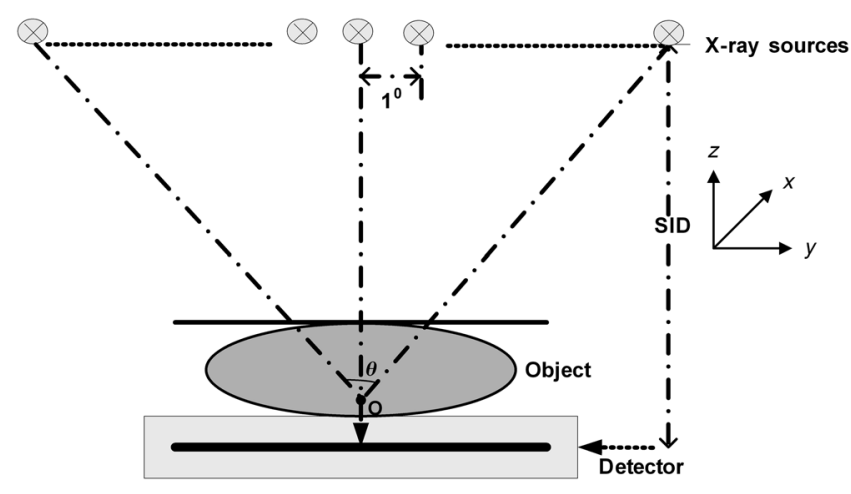

Fig. 1 Illustration of the parallel breast tomosynthesis imaging geometry. 

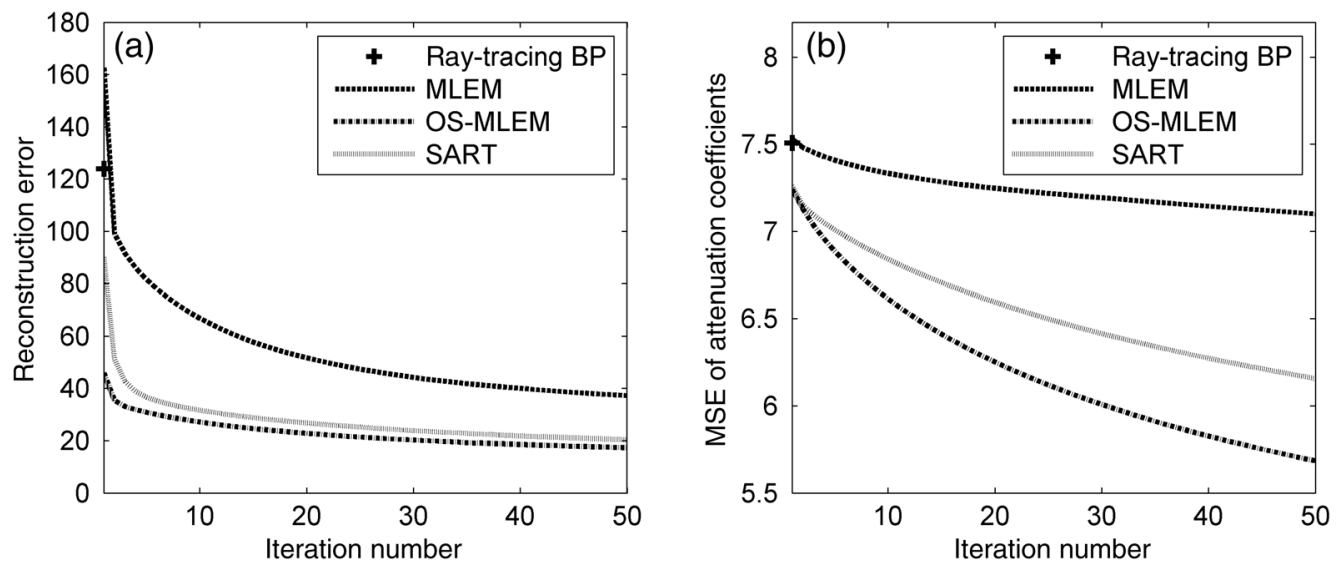

Fig. 2 (a) Attenuation error [Eq. (6)] and (b) mean square error (MSE) of attenuation coefficients [Eq. (7)].

for all the voxels $j . \mu_{j}$ is the exact attenuation coefficient in the FEM model, and $\hat{\mu}_{j}$ is the calculated attenuation coefficient in the reconstruction volume.

\subsection{Phantom Study}

A standard Computerized Imaging Reference Systems, Inc. (CIRS) breast biopsy phantom (CIRS Company; Norfolk, Virginia) was used to evaluate the performance of the reconstruction algorithms. Solid masses and microcalcification clusters were embedded in the phantom as the targets of the evaluation. The four representative algorithms including raytracing BP, MLEM, OS-MLEM, and SART were investigated to generate reconstructed images and to evaluate the image quality of the targets.

In order to evaluate the capability of enhancing the inplane object sharpness and removing interplane blurring, a metrics called the artifact spread function (ASF) was
Ray-tracing BP

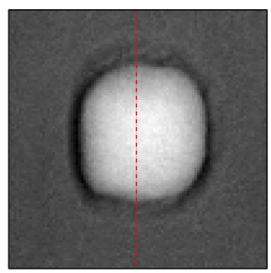

(a)

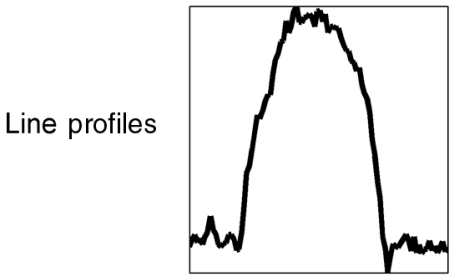

(e)

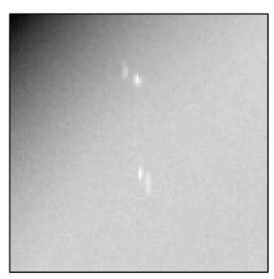

(i)
MLEM

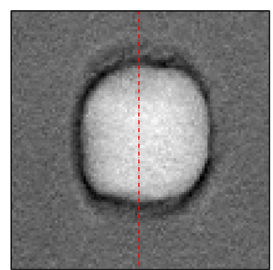

(b)

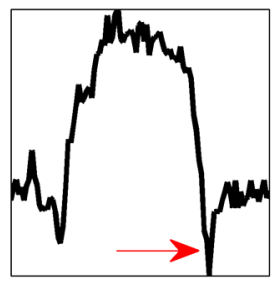

(f)

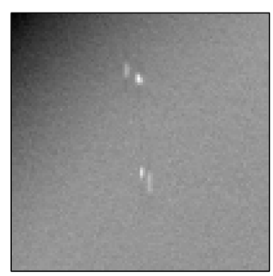

(j)
OS-MLEM

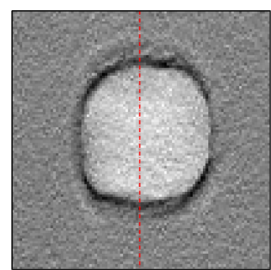

(c)

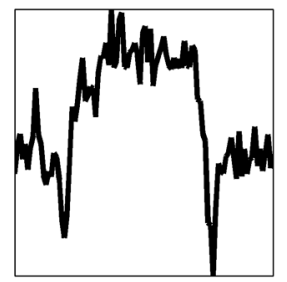

(g)

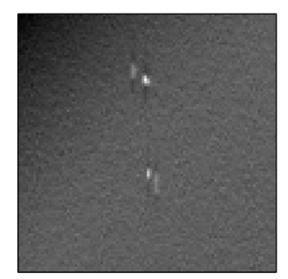

(k)
SART

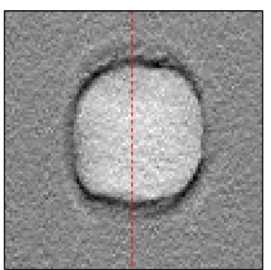

(d)

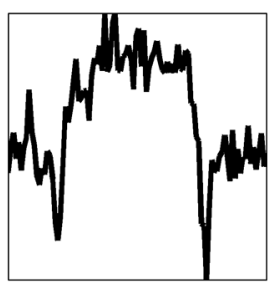

(h)

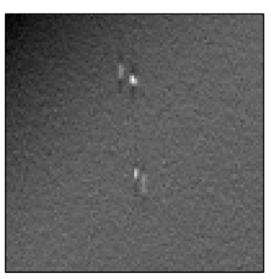

(I)

Fig. 3 Reconstructed regions of interests (ROIs) from the four investigated algorithms: (a)-(d) Masses reconstructed by ray-tracing BP, MLEM, OS-MLEM, and SART. (e)-(h) Line profiles in the reconstructed masses. Line profiles are the pixel intensities along the red lines from the four reconstructed masses. Red arrow is one example of edge enhancement. (i)-(I) Microcalcifications reconstructed by ray-tracing BP, MLEM, OS-MLEM, and SART. 
analyzed for the reconstructed objects. ASF has been used to describe the artifact suppression efficacy along the interplane direction in breast tomosynthesis studies. ${ }^{16}$ It is defined as the ratio of the contrast-to-noise ratio $(\mathrm{CNR})$ values between the off-focus plane and the in-focus plane

$$
\operatorname{ASF}(z)=\frac{\operatorname{CNR}(z)}{\operatorname{CNR}\left(z_{0}\right)},
$$

where $z_{0}$ is the slice location of the in-focus plane of the object and $z$ is the location of the other plane. The CNR value $^{6}$ is defined by

$\mathrm{CNR}=\frac{\mu_{\text {object }}-\mu_{\text {background }}}{\sigma_{\text {background }}}$,

where $\mu_{\text {object }}$ and $\mu_{\text {background }}$ are the mean pixel intensity of the object and image background respectively, and $\sigma_{\text {background }}$ is the root-mean-square noise value of pixel intensity in the image background.

\section{Results}

\subsection{Sphere Simulation}

Figure 2 shows the attenuation error and MSE of attenuation coefficients over the iteration number. As shown in the figure, we found that after the first 10 iterations, attenuation errors dropped from 163.19 to 68.81 (76\% of the overall drop-off) for MLEM, from 45.91 to 27.14 (66\% of the overall drop-off) for OS-MLEM, and from 89.94 to 31.64 (84\% of the overall drop-off) for SART. Accordingly, in the first few iterations, MLEM, OS-MLEM, and SART converged at a fast speed. After all the iterations, OS-MLEM and SART generated the minimal attenuation error with the specified iteration number. Moreover, the attenuation error of the ray-tracing $\mathrm{BP}$ was 129.91 ; therefore, it could be a good initialization for iterative reconstruction algorithms.

\section{Phantom Study}

Figure 3 shows reconstructed regions of interest (ROI) from the four algorithms including ray-tracing BP, MLEM, OSMLEM, and SART, respectively. In Figs 3(a)-3(d) and 3(i)3(1), margins and shapes of the mass and microcalcification reconstructed with all the investigated algorithms can be identified. The microcalcifications are visible although the interplane blurring may reduce their conspicuity and bring difficulties to 3-D localization.

Figures 3(e)-3(h) show the line profiles along the dotted lines in the reconstructed ROIs of the mass. As exampled by the red arrow, the edge enhancement is present in all four reconstructed ROIs, which increases the conspicuity of the mass. Furthermore, the edge enhancement is greater in the ROIs reconstructed by OS-MLEM and SART.

Different algorithms have their implicit or explicit design to remove interplane artifacts. The image background region for noise estimation was chosen as a $40 \times 40$ pixel region far from all target objects and the boundaries in the slice images, and at the same slice as the evaluated object. The mean pixel intensity of a mass was calculated in a $40 \times 40$ pixel region enclosed within the relatively uniform central region of the mass. The selected masses were the same as in Fig. 3. Figure 4 shows the ASF curves for ray-tracing BP, MLEM, OS-MLEM, and SART. CNR drop-offs in the ASF curves are different between the side close to the detector (downside) and the other side close to the x-ray sources (upside). CNR drops $1 \%, 1.5 \%, 2.4 \%$, and $2.5 \%$ on the downside (20 mm from the focus plane) for ray-tracing BP, MLEM, OS-MLEM, and SART, respectively; and it drops 1\%, 1.3\%,

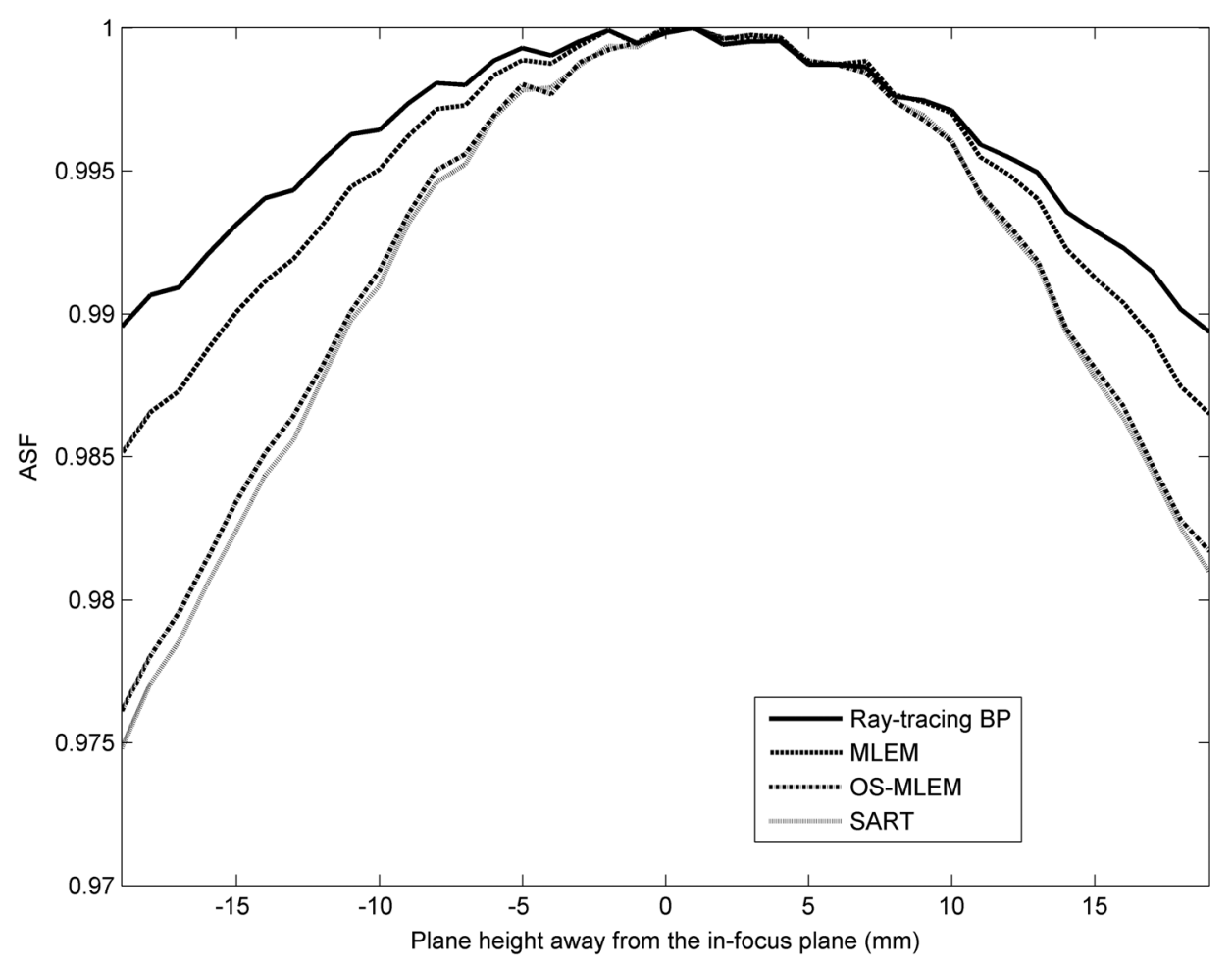

Fig. 4 Artifact spread function (ASF) curves of the investigated reconstruction algorithms. 
$1.8 \%$, and $1.9 \%$ on the upside (20 $\mathrm{mm}$ from the focus plane) for ray-tracing BP, MLEM, OS-MLEM, and SART, respectively. OS-MLEM and SART show bigger CNR drop-offs, so they can remove interplane artifacts better.

\section{Conclusions}

All the investigated ray-tracing-based reconstruction algorithms can provide clear 3-D reconstructed images, and therefore, provide feasible solutions to breast tomosynthesis imaging. OS-MLEM and SART converge faster. Ray-tracing $\mathrm{BP}$ can be a good initialization for iterative reconstruction algorithms.

The phantom study showed that OS-MLEM and SART removed interplane artifacts better based on the ASF curves due to their fast convergence speed.

\section{Acknowledgments}

This work was supported by a research grant from NIH/NCI R01 CA134598-01A1.

\section{References}

1. American Cancer Society, Breast Cancer Facts \& Figures 2013-2014, American Cancer Inc., Atlanta (2013).

2. J. M. Park et al., "Breast tomosynthesis: present considerations and future applications," Radiographics 27(Suppl 1), S231-S240 (2007).

3. M. A. Helvie, "Digital mammography imaging: breast tomosynthesis and advanced applications," Radiol. Clin. North Am. 48(5), 917-929 (2010).

4. J. T. Dobbins, III and D. J. Godfrey, "Digital x-ray tomosynthesis: current state of the art and clinical potential," Phys. Med. Biol. 48, 65-106 (2003).

5. A. Webb, Ed., Introduction to Biomedical Imaging, 1st ed., John Wiley \& Sons, Inc., Hoboken, New Jersey (2002).

6. Y. Zhang et al., "A comparative study of limited-angle cone-beam reconstruction methods for breast tomosynthesis," Med. Phys. 33(10), 3781-3795 (2006).

7. T. Wu et al., "Tomographic mammography using a limited number of low-dose cone-beam projection images," Med. Phys. 30, 365-380 (2003).

8. T. Wu et al., "A comparison of reconstruction algorithms for breast tomosynthesis," Med. Phys. 31, 2636-2647 (2004).
9. W. Zhou, A. Balla, and Y. Chen, "Tomosynthesis reconstruction using an accelerated expectation maximization algorithm with novel data structure based on sparse matrix ray-tracing method," Int. J. Funct. Inform. Personal. Med. 1(4), 355-365 (2008).

10. H. Erdogan, G. Gualtieri, and J. A. Fessler, "Ordered subsets algorithms for transmission tomography," Phys. Med. Biol. 44, 2835-2852 (1999).

11. K. Lange and R. Carson, "EM reconstruction algorithms for emission and transmission tomography," J. Comput. Assist. Tomogr. 8(2), 306316 (1984).

12. K. Lange and J. A. Fessler, "Globally convergent algorithms for maximum a posteriori transmission tomography," IEEE Trans. Image Process. 4(10), 1430-1438 (1995).

13. J. Li et al., "Implementation of an accelerated iterative algorithm for cone-beam SPECT," Phys. Med. Biol. 39, 643-653 (1994).

14. A. C. Kak and M. Slaney, Algebraic Reconstruction Algorithms in Principles of Computerized Tomographic Imaging, Society of Industrial and Applied Mathematics, Philadelphia, PA (2001).

15. G. Yang et al., "Stationary digital breast tomosynthesis system with a multi-beam field emission x-ray source array," Proc. SPIE 6913, 69131A (2008)

16. X. Qian et al., "High resolution stationary digital breast tomosynthesis using distributed carbon nanotube x-ray source array," Med. Phys. 39(4), 2090-2099 (2012).

17. Y. Chen et al., "Breast tomosynthesis reconstruction with a multi-beam x-ray source," Proc. SPIE 7258, 725859 (2009).

18. W. Zhou et al., "Multi-beam x-ray source breast tomosynthesis reconstruction with different algorithms," Proc. SPIE 7622, $76220 \mathrm{H}$ (2010).

19. L. T. G. Guimarães, H. Schiabel, and D. R. M. Stemberg, "Computer simulation in evaluating the attenuation coefficients influence on mammography images contrast," IFMBE Proc. 25(2), 454-457 (2009).

Weihua Zhou obtained his $\mathrm{PhD}$ degree in electrical and computer engineering from Southern Illinois University, Carbondale, Illinois. His research focuses on medical imaging and image processing.

Jianping Lu is a full professor at the University of North Carolina, Chapel Hill, North Carolina.

Otto Zhou is a distinguished full professor at the University of North Carolina, Chapel Hill, North Carolina.

Ying Chen is an associate professor at Southern Illinois University, Carbondale, Illinois. Her research focuses on tomosynthesis, image quality, computer vision, image processing, and analysis. 